\title{
Archéopages
}

Archéopages

Archéologie et société

Hors-série 2 | 2010

Archéologie sans frontières

\section{Umm El Tlel, une exceptionnelle stratigraphie du Paléolithique au Levant}

\author{
Nelly Connet, Éric Boëda et Heba Al Sakhel
}

\section{(2) OpenEdition}

1 Journals

Édition électronique

URL : https://journals.openedition.org/archeopages/746

DOI : 10.4000/archeopages.746

ISSN : 2269-9872

Éditeur

INRAP - Institut national de recherches archéologiques préventives

Édition imprimée

Date de publication : 1 octobre 2010

Pagination : 35-37

ISSN : 1622-8545

\section{Référence électronique}

Nelly Connet, Éric Boëda et Heba Al Sakhel, « Umm El Tlel, une exceptionnelle stratigraphie du

Paléolithique au Levant », Archéopages [En ligne], Hors-série 2 | 2010, mis en ligne le 01 octobre 2010 consulté le 23 février 2023. URL : http://journals.openedition.org/archeopages/746 ; DOI : https:// doi.org/10.4000/archeopages.746 
Buchez N., Midant-Reynes B., 2007, «Le site prédynastique de Kom el-Khilgan (Delta oriental). Données nouvelles sur les processus d'unification culturelle au Iv ${ }^{\mathrm{e}}$ millénaire », Bulletin de l'Institut français d'archéologie orientale du Caire, t. 107, p. 43-70.

Crubezy E. Janin T., Midant-Reynes B, 2002. Adä̈ma II. La Nécropole prédynastique, FIFAO, 47.

Crubezy E., Duschene S., Midant-Reynes B., 2008, La Nécropole d'Adaïma (Haute-Égypte). Présentation générale et implications pour les populations de l'Égypte prédynastique, in Midant-Reynes B., Tristant Y. (ÉD.), Egypt at its origins 2. Origin of the State. Predynastic and Early Dynastic Egypt. Proceedings of the International Conference, Toulouse, 5-8 septembre 2005, p. 289-310.

GuILAINE J.,1994, La Mer partagée. La Méditerranée avant lécriture. 70oo-20oo avant Jésus-Christ, Paris, Hachette, $910 \mathrm{p}$

KAISER W., 1964. «Einige Bemerkungen zur ägyptischen Frühzeit. III. Die Reichseinigung », in Zeitschrift für ägyptische Sprache und Altertumskunde, 91, p. 86-125

KOHLER E.C., 1995, « The state of research on Late Predynastic Egypt: New evidence for the development of the pharaonic state? », in Göttinger Miszellen, 147, p. 79-92.

Newton C., Midant-Reynes B., 2007, «Environmental change and settlement shifts in Upper Egypt during the Predynastic; charcoal analysis at Adaïma ", in The Holocene, 17(8), p. 1109-1118.

Petrie W. M. F., 1921. Corpus of Prehistoric Pottery and Palettes, BSAE \& ERA, 32. Londres

VAN DEN BRINK E.C.M., 1989, «A transitional Late Predynastic - Early Dynastic settlement site in the Northeastern Nile Delta, Egypt», in Mitteilungen des Deutschen Archäologischen Institut Abteilung Kairo,

\section{Umm El Tlel, une exceptionnelle stratigraphie du Paléolithique au Levant}

\author{
Nelly Connet \\ Inrap \\ Éric Boëda \\ Université de Paris X - Nanterre \\ Heba Al Sakhel \\ Conservatrice DGAM
}

e site d'Umm el-Tlel se situe en Syrie, dans la cuvette d'el-Kowm, entre Palmyre et l'Euphrate, à moins de $100 \mathrm{~km}$ au nord-est de Palmyre [Fig.1]. Il est mentionné à de nombreuses reprises dans la littérature dès la fin des années 1960 (Buccellati, 1967 ; Suzuki, Kobori, 1970). Il fut découvert et brièvement étudié par la mission permanente d'el-Kowm en 1978 (Cauvin et al., 1979); le site a de nouveau été signalé par P. Sanlaville, J. Besançon, L. Copeland, F. Hours et S. Muhesen (Besançon et al., 1981, 1982) à l'occasion de prospections dans le cadre de la MPK et de la RCP 438 du CNRS. En 1987 et 1989, il fit l'objet de sondages, sous la direction de M. Molist, M. C. Cauvin et A. Taha, visant essentiellement la partie supérieure de la séquence (Romain, Néolithique et Épipaléolithique). Cest à loccasion de ces sondages qu'une partie 45 (1989), p. 55-108.

et notamment pour le Paléolithique supérieur. En effet, avec 40 niveaux archéologiques successifs, la séquence du Paléolithique supérieur d’Umm el-Tlel, est à ce jour l'une des plus importantes du Proche-Orient. Sa position continentale lui confère une place singulière, ouverte sur le MoyenOrient et l'Eurasie, en retrait de la zone côtière qui concentre la majorité des sites connus du Paléolithique supérieur. Les niveaux du Paléolithique supérieur représentent plus de $5 \mathrm{~m}$ de stratigraphie où se succèdent des occupations sur des séquences longues jalonnées de quelques hiatus.

Après la première approche stratigraphique de reconnaissance d'Éric Boëda et Sultan Muhensen, en 1990, les sept campagnes de fouille de 1991 à 1997 menées par Sylvie Ploux ont permis d'édifier la séquence chrono-culturelle du Paléolithique supérieur d'Umm el-Tlel dans les secteurs 2 et 5 du site (Ploux, Soriano, 2003). Le Paléolithique supérieur est fouillé sur deux secteurs du site, avec un Aurignacien ancien dans la partie sud et une séquence allant de l'Ahmarien au Kébarien dans la partie nord.

C'est sur cette dernière que nos travaux se sont concentrés entre 2005 et 2007 , avec une fouille ponctuelle des niveaux anciens et une fouille extensive des niveaux kébariens. En 2005, la reprise des travaux sur le Paléolithique supérieur a été motivée par l'extraordinaire potentiel de recherche que représente une telle séquence chrono-culturelle mise en lumière par les premières études dirigées par S. Ploux, malheureusement disparue prématurément. Après une première étape de reconnaissance verticale de la séquence, il est apparu nécessaire d'explorer chaque niveau par une fouille extensive; d'une part pour recueillir du mobilier afin d'affiner le diagnostic techno-culturel de certains niveaux difficiles à caractériser; d'autre part pour entreprendre une lecture planimétrique des occupations et en comprendre, si possible, les modalités d'occupation.

En 2005 et 2006, l'ouverture des niveaux aurignaciens II $2 a$ à II 2 c sur une petite surface et les premières observations effectuées par Marie-Agnès Courty (CNRS) ont permis d'identifier des traces d'aménagements pouvant s'apparenter à des trous de piquet. Ces premiers résultats sont encourageants et permettent d'envisager, à une plus grande échelle, une lecture de l'organisation spatiale des différents niveaux d'occupation et de leur possible évolution de statut et de fonction.

En 2006 et 2007, après une reconnaissance de de la séquence aurignacienne fut mise au jour (Molist, Cauvin, 1990 ; Molist et al., 1990). En 1987, un sondage a également été réalisé dans les niveaux du Paléolithique moyen, à la périphérie immédiate du puits, par A. Vincent. De 1991 à 2005, les fouilles se firent sous la responsabilité d'Éric Boëda et de S. Muhesen (DGAm Damas) et depuis 2006 sous la responsabilité d'Éric Boëda et d'Heba Al Sakhel. L'étude du Paléolithique supérieur a été assurée par S. Ploux (CNRS) de 1991 à 1997. Nelly Connet est intervenue à partir de 2005 comme responsable de fouille du secteur 2 (Paléolithique supérieur) [Fig.1].

La séquence du site d'Umm el-Tlel est exceptionnelle pour la Préhistoire du Levant la stratigraphie du complexe I, nous avons entrepris la fouille extensive des niveaux kébariens, dirigée depuis 2007 par Hazar Al Ahmar. Six niveaux principaux ont été identifiés dans la partie nord du secteur 2 et c'est une surface d'un seul tenant de près de $20 \mathrm{~m}^{2}$ qu'il sera possible d'investir dans les années à venir. Le mobilier épipaléolithique mis au jour est étudié par Hazar Al Ahmar dans le cadre d'un doctorat de l'université de Paris X sur « l'Épipaléolithique dans les régions arides désertiques et semi-désertiques du Levant». Cette dernière est en charge, depuis 2007 , de la fouille des niveaux épipaléolithiques sur ce secteur du site. 


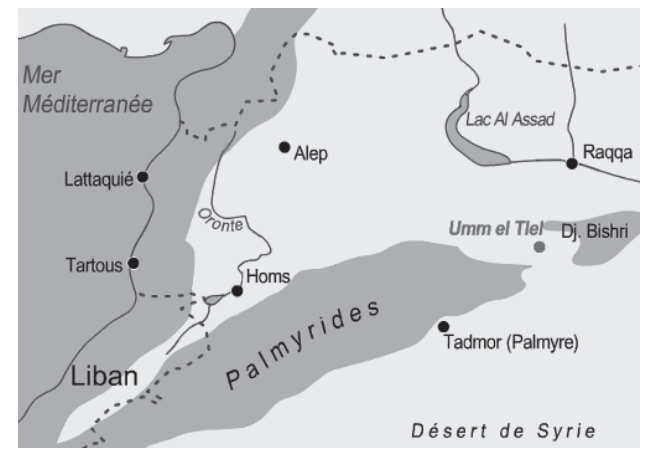

$\mathscr{\infty}$

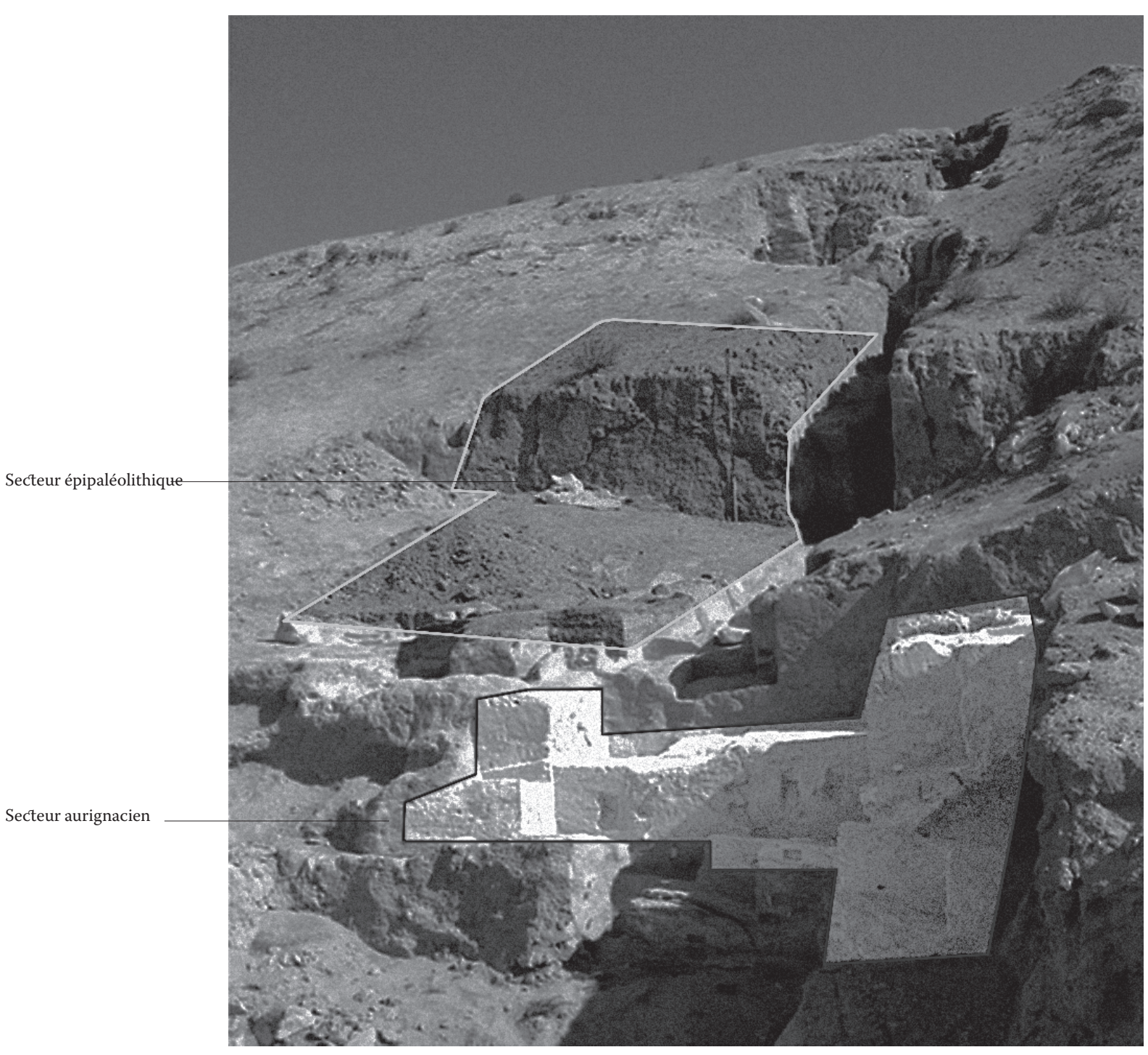

[Fig. 1] Zones de travail dans le secteur 2 depuis 2004. 
Nous projetons l'ouverture du complexe D du secteur 2 afin de caractériser les industries encore mal connues à la charnière des Aurignaciens/ Ahmariens et de l'Épipaléolithique.

BOËDA É., BONILAURI S. 2006 : « The intermediate paleolithic: the first bladelet production 40.00o years ago ", Anthropologie, XLIV/1, p. 75-92. Ploux S., Soriano S., 2003 : «Umm el Tlel, Une séquence du paléolithique supérieur en Syrie centrale. Industries lithiques et chronologie culturelle» Paléorient, vol. 29/2, p. 5-34, CNRS Éditions.

Suzuki H., Kobori I., 1970, « Report of the Reconnaissance Survey on Palaeolithic Sites in Lebanon and Syria », Bulletin, I, Tokyo, University Museum.

\section{Archéologie du christianisme éthiopien : quinze ans de collaboration scientifique entre le Centre français d'études éthiopiennes et l'Inrap}

\author{
Marie-Laure Derat \\ CNRS, Centre francais détudes éthiopiennes, Addis-Abeba (USR 3137) \\ François-Xavier Fauvelle-Aymar \\ CNRS, TRACES-UMR 5608, Toulouse, et chercheur honoraire \\ à la School of Geography, Archaeology and Environmental Studies \\ de l'université du Witwatersrand (Johannesburg, Afrique du Sud) \\ Anne-Marie Jouquand \\ Inrap, UMR 6173 CITERES « Laboratoire archéologie et territoire» \\ Bertrand Poissonnier \\ Inrap
}

D epuis une quinzaine d'années, des recherches archéologiques conduites sous l'égide du Centre français d'études éthiopiennes (CFEE) ont contribué à revisiter le christianisme éthiopien et son contexte géographique et historique. De nombreux archéologues de l'Afan puis de l'Inrap ont participé à ces recherches, qui sont désormais inscrites dans le cadre d'un partenariat scientifique. D'autres collaborations ont également été mises en place au cours des dernières années, notamment au sujet de la préhistoire récente de la Corne de l'Afrique et dans le champ de recherche de l'islam ancien, encore relativement neuf dans cette région du monde ${ }^{2}$. Mais les pages qui suivent ont surtout pour objectif de mettre en relief l'apport de la collaboration de longue haleine pratiquée entre chercheurs de diverses disciplines et institutions sur le terrain spécifique de l'Éthiopie chrétienne médiévale.

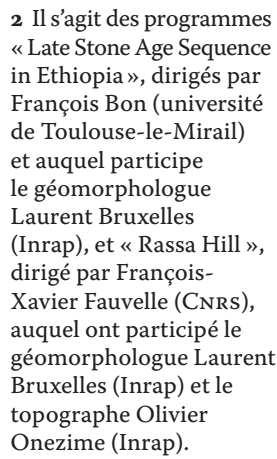

Les lacunes documentaires du christianisme éthiopien. Christianisée au IV ${ }^{\mathrm{e}}$ siècle, l'Éthiopie est sans conteste l'une des régions du monde ayant connu la plus longue tradition chrétienne continue, puisque c'est toujours dans cette tradition de christianisme monophysite (historiquement liée à l'Église copte d'Égypte) que vivent la majorité des habitants des hauts plateaux. Et pourtant le christianisme éthiopien souffre de sérieuses lacunes documentaires. Ainsi est-il remarquable que la considérable documentation manuscrite conservée dans les monastères du pays se rapporte, à de très rares exceptions près, à la période postérieure au XIII ${ }^{\mathrm{e}}$ siècle, faisant suite à l'apparition de la dynastie royale des Salomoniens. Quant à l'archéologie des périodes médiévales et modernes, elle présente un tableau mitigé : très peu de sites à vocation liturgique ont été fouillés, principalement pour la raison qu'une tradition vivante continue généralement de les animer et les rend inaccessibles à des investigations poussées (les études de l'architecture et des peintures posent moins de problème à cet égard), et aucun site « civil » n'a encore été identifié sur le terrain pour la période antérieure à la fixation des rois à Gondar (XVII ${ }^{\mathrm{e}}$-XVIII ${ }^{\mathrm{e}}$ siècles). Il est ainsi paradoxal que la période des $\mathrm{XIII}^{\mathrm{e}}-\mathrm{XV}^{\mathrm{e}}$ siècles, période de formidable dynamisme du monachisme éthiopien et d'expansion du royaume chrétien, ne puisse encore faire l'objet d'une approche nourrie d'archéologie. Durant cette période, le camp royal est itinérant. Le roi et sa cour, sans doute plusieurs milliers de personnes, composée de dignitaires civils et religieux, d'hommes de guerres, de serviteurs et d'esclaves, se déplacent presque chaque année au gré des guerres et des nécessités matérielles (nourriture et bois) dans un endroit différent pour la saison des pluies. On possède plusieurs sources qui décrivent ce camp, mais sans doute la description de Francisco Alvares, qui fait partie de l'ambassade portugaise dans l'Abyssinie des années 1520-1526, est-elle l'une des plus vivantes. Ces déplacements sur un vaste territoire s'accompagnent de créations d'églises et de monastères organisés en réseaux, sur lesquels s'appuie le pouvoir royal et qui sont mentionnés dans les sources écrites sans que l'on sache toujours les localiser précisément.

Pour la période antérieure, celle que l'on pourrait appeler le « Haut-Moyen Âge » éthiopien, la situation documentaire est encore plus critique. Les sociétés d'Éthiopie ne sont alors connues que par quelques mentions dans des sources extérieures, chrétiennes d'Égypte ou musulmanes, et seuls de rares témoins de cette période, architecturaux, archéologiques ou de la culture matérielle, ont survécu. C'est en somme pour la période de l'Antiquité tardive ( $\mathrm{IV}^{\mathrm{e}}-\mathrm{VIII}{ }^{\mathrm{e}}$ siècles) que nous sommes le mieux renseignés : sources externes (grecques le plus souvent), séries numismatiques et inscriptions (en grec, en geez ou en sud-arabique) nous informent sur les noms, titres et faits de quelques souverains au moins nominalement chrétiens des $\mathrm{IV}^{\mathrm{e}}-\mathrm{VI}^{\mathrm{e}}$ siècles, tandis que plusieurs églises fouillées sur les territoires éthiopien ou érythréen attestent l'existence d'une multiplicité 